PoS $\quad \begin{aligned} & \text { PROCEEDINGS } \\ & \text { OF SCIENCE }\end{aligned}$

\title{
Exotic decays of the Higgs boson
}

\author{
María Cepeda*† \\ CERN \\ E-mail: cepeda@cern.ch
}

Summary of the direct searches for exotic decays of $\mathrm{h}(125)$ performed by the CMS experiment.

38th International Conference on High Energy Physics

3-10 August 2016

Chicago, USA

*Speaker.

${ }^{\dagger}$ On behalf of the CMS Collaboration. 
The ATLAS and CMS experiments announced in 2012 the discovery of the Higgs boson $\mathrm{h}(125)$, a spin- 0 particle with a mass of $125 \mathrm{GeV}[1,2]$. Determining its couplings and studying its compatibility with the Standard Model (SM) predictions is one of the main tasks of particle physics today. It is essential to discern if the discovered boson is in fact the SM Higgs, or if a door to new physics opens from the Higgs Sector. While the results obtained so far by the LHC experiments show good agreement with the Standard Model predictions, the 95\%CL upper limit on Beyond the Standard Model (BSM) decays of the boson is currently as large as 34\% [3], and indirect couplings measurements are not expected to constrain it beyond $\mathscr{O}(5-10) \%$. There is therefore a strong motivation to perform direct searches targeting specific exotic $\mathrm{h}(125)$ decays.

This article briefly summarises the key results obtained by CMS in two different search areas: $\mathrm{h}(125)$ decays to pseudoscalars and scalars, and $\mathrm{h}(125)$ decays to lepton-flavour-violating lepton pairs. Other BSM decays of the $125 \mathrm{GeV}$ Higgs boson, such as decays to invisible particles, are not covered. The data samples used were collected by CMS in 2012 and 2015, and correspond to an integrated luminosity of approximately $20 \mathrm{fb}^{-1}$ at a center-of-mass energy of $8 \mathrm{TeV}$ and $2.6 \mathrm{fb}^{-1}$ at a center-of-mass energy of $13 \mathrm{TeV}$. The CMS detector is described in detail in [4].

\section{1. $\mathbf{h}(125) \rightarrow$ aa}

Among the proposed extensions of the SM including scalar sectors are the two-Higgs doublet models (2HDM). The 2HDM incorporate two doublet fields, that after symmetry breaking lead to five physical states: two scalars, one pseudoscalar, and two charged Higgs bosons. 2HDM models are classified in four types depending on the doublet each type of fermions couples to. Types 1 and 2, in which the leptons have the same couplings as the down-type quarks, are the most commonly considered. Involving an additional EWK scalar signal we can expand to a $2 \mathrm{HDM}+\mathrm{S}$ model, in which we have two extra singlet states: a scalar and a pseudoscalar, both possibly light. The NextTo-Minimal-Supersymmetric-Standard-Model (NMSSM) is a particularly strong motivated case of 2HDM+S type-2. Other BSM theories also motivates the search for a low-mass Higgs boson, such as dark-SUSY models.

In this context, CMS has performed different searches for a SM-like Higgs boson decaying to pairs of lighter scalars or pseudoscalars in final states involving $\tau$ leptons, muons, and $\mathrm{b}$ quarks. In this proceeding the symbol $a$ refers to both the light scalar and pseudoscalar. Light boson masses from $0.25 \mathrm{GeV}$ up to one half of the mass of $\mathrm{h}(125)$ are probed. The searches were performed with $\mathrm{fb}^{-1}$ of data collected by the CMS experiment in 2012 at a center-of-mass energy of $8 \mathrm{TeV}$.

\section{$1.1 \mathrm{~h}(125) \rightarrow \mathrm{aa} \rightarrow 4 \mu$}

The first of the searches summarised in this document contemplates final states with four muons [5], and probes a very low mass range for $a$, between $0.25-3.55 \mathrm{GeV}$. It takes advantage of the excellent muon resolution of CMS. The interpretation of the result is given in two different scenarios: a NMSSM interpretation in which each pair of muons originates in the decay of a light pseudoscalar $a_{1}$, and a dark-SuSy model in which they arise from $\gamma_{D}$ produced in the decay of a neutralino $\left(h \rightarrow n_{1} n_{1}, n_{1} \rightarrow n_{D} \gamma_{D}, \gamma_{D} \rightarrow \mu \mu\right.$ ). The analysis strategy exploits the 2D dimuon mass plane, minimising the background by selecting events with $m_{1 \mu \mu} \approx m_{2 \mu \mu}$. Data driven techniques are used to determine the backgrounds, mainly coming from $b \bar{b}$ and $\mathrm{J} / \Psi$ processes. No excess 
over the expected background contribution is observed, and the results are given both as a model independent 95\% upper limits on the product of the cross section times branching ratio of a hypothetical $a$ state, and as a model dependent interpretation in the two scenarios mentioned (NMSSM and dark-SuSy).

\section{$1.2 \mathrm{~h}(\mathbf{1 2 5}) \rightarrow \mathrm{aa} \rightarrow \mathbf{4} \tau$}

Two complementary analyses $[6,7]$ target decays of $\mathrm{h}(125)$ to a pair of light scalars decaying to two pairs of highly boosted tau leptons. Both analysis exploit the presence in the signal topology of two well-separated same-sign muons, each coming from the decay of a tau lepton, to highly suppress background contamination coming from Standard Model processes. The main background, misidentified taus appearing in QCD processes, is modelled from data in both cases. The first one of the analysis identifies the two pairs of boosted taus by combining each muon with a opposite-charge track located nearby. This technique probes a reasonably low mass range for the muon-track pair (4-8 GeV). Signal extraction is performed through a maximum-likelihood fit to the 2D distribution of $m_{1 \mu}$-track versus $m_{2} \mu$ track. The second analysis probes a higher mass range (5-15 GeV), looking for the combination of a muon and a tau decaying hadronically (boosted $\mu \tau$ pair). The analysis is sensitive to the gluon fusion (GF), $\mathrm{W}$ and $\mathrm{Z}$ associated (VH), and vector boson fusion (VBF) production channels for the Higgs. VH decays are enhanced by selecting events with an additional muon that yields high transverse mass when combined with the missing energy in the event. In the GGF and VBF production modes this second muon arises from a tau lepton decay. Neither analysis observes a excess of events, and upper limits are set on the cross section times branching ratio and on the branching ratio for the considered process.

\section{$1.3 \mathrm{~h}(125) \rightarrow \mathrm{aa} \rightarrow 2 \mu 2 \tau$ and $\mathrm{h}(125) \rightarrow \mathrm{aa} \rightarrow 2 \mu 2 \mathrm{~b}$}

The two final searches follow a similar analysis strategy. They focus respectively on $\mathrm{h}(125)$ decays into a dimuon pair and a ditau pair [8] and h(125) decays into a dimuon pair and a pair of b quarks [9], and target a general $2 \mathrm{HDM}+\mathrm{S}$ scalar scenario in which the higgs decays to a pair of new light bosons a. The analysis involving a ditau pair is categorized depending on the decay of the tau leptons: $\tau_{e} \tau_{e}, \tau_{h} \tau_{h}, \tau_{e} \tau_{\mu}, \tau_{e} \tau_{h}, \tau_{\mu} \tau_{h}$ final states are taken into account. In both cases a maximum likelihood fit to the dimuon mass distribution is performed to discriminate signal and background, exploiting the good mass resolution provided by CMS. Background is reduced by ensuring compatibility of the masses of the two $a$ candidates. Masses of the light boson between 20 and $62.5 \mathrm{GeV}$ are probed. No statistically significant excess of events is observed and upper limits are set on the cross section times branching ratio, and on the branching ratio itself.

\subsection{Comparison of reach of the different $h(125) \rightarrow$ aa decay modes}

No significant excess is found in any of the decay modes studied. The results of the different searches for decays of the standard model like Higgs boson to a pair of light bosons can then be interpreted in a coherent manner, exploiting the complementarity of their mass coverage to compare the results to the predictions of different $2 \mathrm{HDM}$ scenarios with an additional scalar singlet in a large phase-space. In Ref [10] such comparison is shown for type 1, 2, 3 and 4 scenarios, for different values of $\tan \beta$. 
Figure 1 (left) shows the observed 95\% CL exclusion limits on $\sigma(h) / \sigma_{S M} \times \mathscr{B}(h(125) \rightarrow$ $\mu \mu) \times\left(a \rightarrow \mu \mu^{2}\right)$ within the contest of the $2 \mathrm{HDM}+\mathrm{S}$ model type- 1 and type-2, comparing the different searches presented, and assuming that the branching fractions of the pseudoscalar boson to muons and to tau leptons follows the relationship $\frac{\Gamma(a \rightarrow \mu \mu)}{\Gamma(a \rightarrow \tau \tau)}=\frac{m_{\mu}^{2} \sqrt{1-\left(2 m_{\mu} / m_{a}\right)^{2}}}{m_{\tau}^{2} \sqrt{1-\left(2 m_{\tau} / m_{a}\right)^{2}}}$. For type-1 and type- 2 there is no dependence on $\tan \beta$ for the branching fractions of the pseudoscalar boson to muons and $b$ quarks.

Figure 1 (right) shows the $95 \% \mathrm{CL}$ on $\sigma(h) / \sigma_{S M} \times \mathscr{B}(h(125) \rightarrow a a)$ the $2 \mathrm{HDM}+\mathrm{S}$ type-3 for different values of $\tan \beta$ for a pseudoscalar mass $m_{a}=40 \mathrm{GeV}$, for the $\mathrm{h}(125) \rightarrow$ aa $\rightarrow 2 \mu 2 \tau$ and $\mathrm{h}(125) \rightarrow \mathrm{aa} \rightarrow 2 \mu 2 \mathrm{~b}$ searches. The branching fractions of the pseudoscalar boson to SM particles are computed following the prescriptions in Ref.[11].
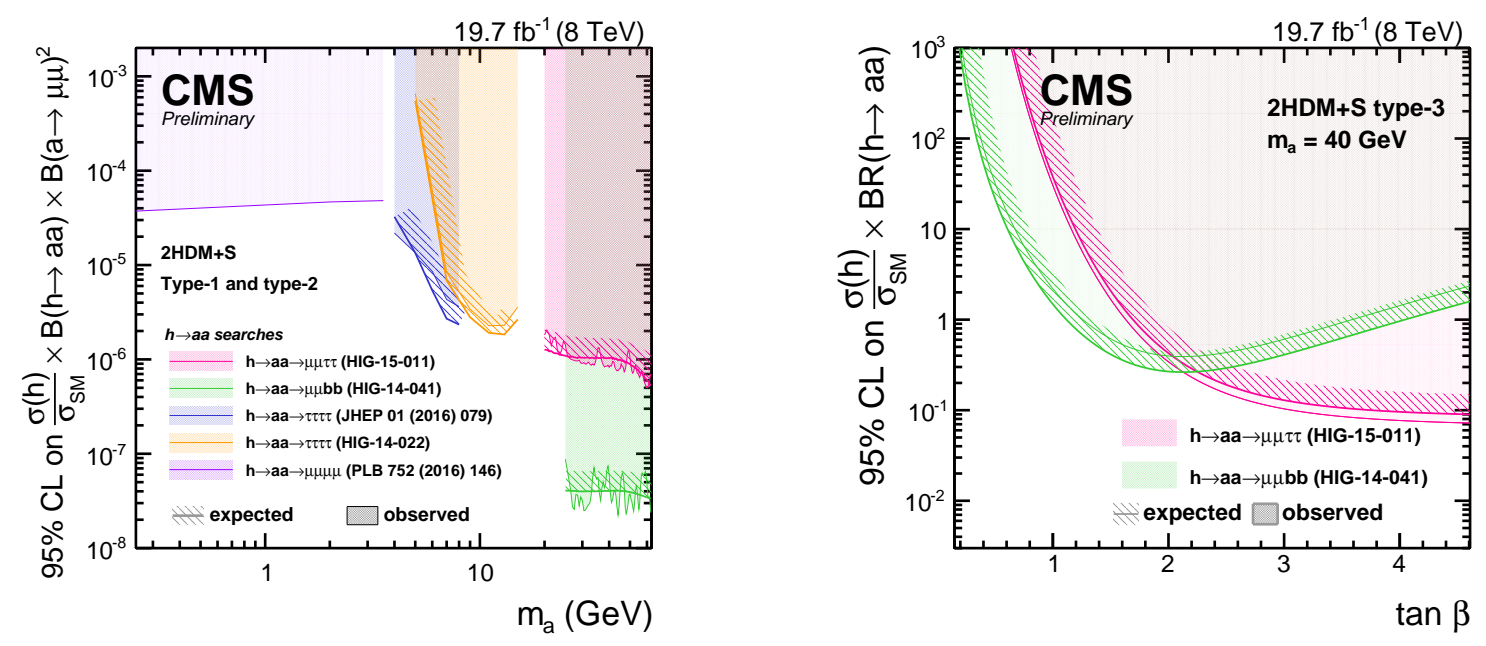

Figure 1: Left: $95 \% \mathrm{CL}$ exclusion limits on $\sigma(h) / \sigma_{S M} \times \mathscr{B}(h(125) \rightarrow \mu \mu) \times\left(a \rightarrow \mu \mu^{2}\right)$ in 2HDM+S type-1 and type-2 . Right: $95 \% \mathrm{CL}$ on $\sigma(h) / \sigma_{S M} \times \mathscr{B}(h(125) \rightarrow a a)$ in $2 \mathrm{HDM}+\mathrm{S}$ type-3.

\section{Lepton Flavour Violating decays of $h(125)$}

In the SM, lepton-flavour-violating (LFV) decays of the Higgs boson are forbidden if the theory is to be renormalizable. If this requirement is relaxed, so the theory is valid only to a finite energy scale, then LFV couplings may be introduced. The CMS experiment performed the first direct search for LFV decays of the Higgs to a $\mu \tau$ pair [12], at a center-of-mass energy of $8 \mathrm{TeV}$, followed by searches for the decay to $e \tau$ pairs and $e \mu$ pairs at $8 \mathrm{TeV}$ [13]. The $\mu \tau$ search was also performed with the $13 \mathrm{TeV}$ dataset collected in 2015 [14], yet with smaller sensitivity due to the limited amount of data ( $2.3 \mathrm{fb}^{-1}$ at $13 \mathrm{TeV}$ compared to $20 \mathrm{fb}^{-1}$ at $8 \mathrm{TeV}$ ).

The searches for $\mathrm{H} \rightarrow \mu \tau$ and $\mathrm{H} \rightarrow e \tau$ decays follow a similar strategy. The final states considered are $\mu \tau_{h}, \mu \tau_{\mathrm{e}}, \mathrm{e} \tau_{h}, \mathrm{e} \tau_{\mu}$. The analysis strategy is based on a categorization based in the number of jets in the event, to discriminate the GF and VBF production modes. The hardness of the $p_{T}$ spectrum of the muon (electron), which decays promptly from the Higgs, and the different angular correlations observed between the leptons and the $\vec{E}_{T}^{\text {miss }}$ provide discrimination with respect to the 


\begin{tabular}{cccc}
\hline \hline \multirow{2}{*}{ Decay } & \multirow{2}{*}{ Dataset } & \multicolumn{2}{c}{ 95\% CL upper limit } \\
& & $\mathrm{BR}\left(\mathrm{h}(125) \rightarrow l_{\alpha} l_{\beta}\right)$ & $\sqrt{\left|Y_{l_{\alpha} l_{\beta}}\right|^{2}+\mid Y_{\left.l_{\beta} l_{\alpha}\right|^{2}}}$ \\
\hline \hline$\mu \mathrm{e}$ & $8 \mathrm{TeV}$ & $0.36 \cdot 10^{-3}\left(0.48 \cdot 10^{-3}\right)$ & $5.4 \cdot 10^{-4}$ \\
\hline $\mathrm{e} \tau$ & $8 \mathrm{TeV}$ & $0.69(0.75) \%$ & 0.0024 \\
\hline \multirow{2}{*}{$\mu \tau$} & $8 \mathrm{TeV}$ & $1.51(0.75) \%$ & 0.0036 \\
& $13 \mathrm{TeV}$ & $1.20(1.62) \%$ & 0.0032 \\
\hline \hline
\end{tabular}

Table 1: Upper limits on the branching fraction of $\mathrm{H} \rightarrow e \mu, \mathrm{H} \rightarrow e \tau$ and $\mathrm{H} \rightarrow \mu \tau$, and the corresponding upper limits on the off-diagonal $\sqrt{\left|Y_{l_{\alpha} l_{\beta}}\right|^{2}+\left|Y_{l_{\beta} l_{\alpha}}\right|^{2}}$ Yukawa couplings

$\mathrm{SM} \mathrm{H} \rightarrow \tau \tau$ and the $\mathrm{Z} \rightarrow \tau \tau$ processes. Other background contributions come from the production of $t \bar{t}$ and diboson events, as well as from misidentified leptons in $\mathrm{W}+$ jets and multijet events.

The search for $\mathrm{H} \rightarrow \mu e$ decays employs a categorization strategy based on the kinematic properties of the leptons and the different resolution of central and forward lepton reconstruction to increase the sensitivity of the search. VBF production is targeted through dedicated jet categories.

A slight excess of signal events with a significance of $2.4 \sigma$, which corresponds to a best fit for the branching ratio of $\mathrm{H} \rightarrow \mu \tau=0.84_{-0.37}^{+0.39} \%$ was observed in the $\mathrm{H} \rightarrow \mu \tau$ case in the $8 \mathrm{TeV}$ analysis. The analysis of the $201513 \mathrm{TeV} \mu \tau$ dataset shows no deviation with respect to the SM background. The analysis of the full 2016 dataset is necessary to make definitive conclusions on the excess.

No deviation from the SM background was observed for the $\mathrm{H} \rightarrow \mathrm{e} \tau$ and $\mathrm{H} \rightarrow \mathrm{e} \mu$ decays. 95\% upper limits on the branching ratio are set in all cases, and subsequently used to constrain the off-diagonal Yukawa couplings of the boson. They are summarized in Table 1.

\subsection{Conclusions}

The CMS collaboration published a comprehensive set of production and decay measurements of the new SM-like Higgs boson using the data collected in the Run I of the LHC, and it is currently in the process of analysing the Run II dataset to broaden the reach of these studies. Searches in rarer modes are already sensitive enough for a potential discovery.

Different searches for $\mathrm{h}(125)$ decays to low-mass scalars and pseudoscalars have been performed in the context of the $2 \mathrm{HDM}+\mathrm{S}$ and NMSSM models with the Run I dataset. They involve a wide spectra of decay modes involving b quarks, muons, and tau leptons, and covering a wide mass range for the scalar mass, from $0.25 \mathrm{GeV}$ to $62.5 \mathrm{GeV}$. No deviation from the Standard Model prediction is observed, and a coherent interpretation in terms of the $2 \mathrm{HDM}+\mathrm{S}$ model is provided.

The first direct searches for lepton-flavour-violating decays of the Higgs boson in the $\mu \tau$, e $\tau$ and $\mathrm{e} \mu$ channels are also presented. A slight excess of signal events with a significance of $2.4 \sigma$, was observed in the $\mathrm{H} \rightarrow \mu \tau$ case in the $8 \mathrm{TeV}$ analysis. No deviation from the SM background was observed for the $\mathrm{H} \rightarrow \mathrm{e} \tau$ and $\mathrm{H} \rightarrow \mathrm{e} \mu$ decays. 


\section{References}

[1] ATLAS Collaboration, "Observation of a new particle in the search for the Standard Model Higgs boson with the ATLAS detector at the LHC”, Phys. Lett. B 716 (2012) 1, [arXiv:1207.7214].

[2] CMS Collaboration, "Observation of a new boson with mass near $125 \mathrm{GeV}$ in pp collisions at $\sqrt{s}=7$ and 8 TeV”, JHEP 1306 (2013) 081, [arXiv:1303.4571].

[3] ATLAS and CMS Collaborations, "Measurements of the Higgs boson production and decay rates and constraints on its couplings from a combined ATLAS and CMS analysis of the LHC pp collision data at 7 and 8 TeV”, J. High Energy Phys. 08 (2016) 045, [arXiv:1606.02266]

[4] CMS Collaboration, “The CMS experiment at the CERN LHC”, JINST 3 (2008) S08004.

[5] CMS Collaboration, "A search for pair production of new light bosons decaying into muons", Phys. Lett. B 752 (2016) 146, [arXiv:1506.00424]

[6] CMS Collaboration, "Search for a very light NMSSM Higgs boson produced in decays of the 125 GeV scalar boson and decaying into $\tau$ leptons in pp collisions at $8 \mathrm{TeV}$ ", JHEP 01 (2016) 079, [arXiv:1510.06534]

[7] CMS Collaboration, "Search for Higgs decays to new light bosons in boosted tau final states", CMS-PAS-HIG-14-022

[8] CMS Collaboration, "Search for the exotic decay of the Higgs boson to two light pseudoscalar bosons with two taus and two muons in the final state at 8 TeV", CMS-PAS-HIG-15-011

[9] CMS Collaboration, "Search for exotic decays of the Higgs boson to a pair of new light bosons with two muon and two b jets in final states", CMS-PAS-HIG-14-041

[10] CMS Collaboration, "Search for light bosons in decays of the $125 \mathrm{GeV}$ Higgs boson in proton-proton collisions at $8 \mathrm{TeV}$ ”, Submitted to JHEP, [arXiv:1701.02032]

[11] D. Curtin et al., "Exotic decays of the 125 GeV Higgs boson", Phys. Rev. D 90 (2014) 075004, [arXiv:1312.4992]

[12] CMS Collaboration, "Search for Lepton-Flavour-Violating Decays of the Higgs Boson”, Phys. Lett. B 749 (2015) 337, [arXiv:1502.07400].

[13] CMS Collaboration, "Search for lepton flavour violating decays of the Higgs boson to $e \tau$ and $e \mu$ in proton proton collisions at $\sqrt{s}=8 \mathrm{TeV}$ ", Phys. Lett. B 763 (2016) 472, [arXiv:1607.03561].

[14] CMS Collaboration, "Search for Lepton-Flavour-Violating Decays of the Higgs Boson", CMS-PAS-HIG-16-005 